\title{
The relationship of antibody levels to the clinical spectrum of human neurocysticercosis
}

\author{
D Zini, V J R Farrell, A A Wadee
}

\begin{abstract}
One hundred proven cases of cerebral cysticercosis were studied with an enzyme linked immunoassay (ELISA) employing cyst fluid as antigen, with a view to detecting specific antibodies in serum and cerebrospinal fluid (CSF). Antibody levels were correlated with the clinical presentation of the patients, the type and number of cysts detected on their brain scans, the anatomical position of these cysts and the presence of lymphocytes in the CSF. Patients could be divided into two distinct categories, one with low levels of antibody in the serum and absent antibody in the CSF, and the other with high levels in both the serum and the CSF. This differentiation matched the clinical presentation of a benign and a malignant group. Antibody levels could not be related to the type of cysts as observed on the brain scan, but depended on the anatomical position of the cyst, being lower if the cysts were confined to the cerebral cortex. A correlation of antibody levels with the number of cysts was only found in the benign group.
\end{abstract}

Human cysticercosis is caused by the metacestode stage of Taenia solium ( $T$ solium). It is the most common parastic disease affecting the central nervous system, and represents a major health problem in South Africa where there is a high prevalence of this disease in the rural black population. ${ }^{12}$

Although the introduction of computed tomography (CT) has increased the diagnostic accuracy of neurocysticercosis, ${ }^{3}$ serological confirmation is often required to exclude other infective, non-infective or neoplastic lesions of the central nervous system. A reliable and specific serological test for the detection of antibodies in cysticercosis would therefore be of great value in supporting the radiological diagnosis, particularly where advanced imaging techiques are not available. Although a variety of serological tests for cysticercosis have been described ${ }^{3-5}$ most of these have been performed with somatic antigen which appears to lack specificity due to the presence of components reacting against antibodies produced in other helminthic infections. ${ }^{67}$ The use of purified antigen in an enzyme linked immunoassay (ELISA) has considerably increased diagnostic specificity.
However, both the complexity of the purification process and the loss of sensitivity inherent to the use of purified antigen makes this method unsuitable for large scale diagnosis. ${ }^{8}$ In this study an antigen derived from the fluid of $T$ solium was employed in an ELISA which detected significant antibody levels in most serum samples from patients with radiologically proven cysticercosis. It has generally been thought that because of the central nervous system (CNS) distribution of $T$ solium, antibodies would be observed in high titre in cerebrospinal fluid (CSF) from patients with this disease. This study compared antibody titres in the serum and CSF from 100 proven cases of cysticercosis. In addition these antibody titres were related to various other CNS features such as the number, position and stage of development of the cysts; and the lymphocytosis observed in the CSF of many of these cases.

\section{Materials and methods \\ Patients}

One hundred patients with neurocysticercosis were included in the study. Of these, 30 were diagnosed as having the malignant form of the disease and the remaining $\mathbf{7 0}$ were classified as belonging to the benign group. The diagnostic features used to define these two are presented in table 1. Evidence of extra-cerebral calcified cysts could be detected in only two patients on general and radiological examinations. All patients were male between the ages 18-45 and were admitted to one hospital and examined radiologically. Their sera and CSF were submitted for laboratory analysis. None of these patients were under any steroid treatment at the time blood was drawn for these studies.

The malignant group consisted of patients with complex neurological illnesses as listed in table 2. This table also indicates the number of patients used per major diagnostic feature in addition to demonstrating other complex neurological features. Radiological investigations of this group showed the presence of cysts either at the base of the brain or in the major cisterns or within the ventricular system. All of these patients had pleocytosis in the CSF associated with an increased protein in the majority of cases and a decreased sugar content. All had a prolonged illness, frequently resulting in neurological disability. Twenty five of these patients required surgery as a corrective procedure. From the remaining five, two died before operation and two refused surgical treatment. 
Table 1 Diagnostic features of neurocysticercosis in the present study

\begin{tabular}{lll}
\hline Clinical manifestation & Benign $(n=70)$ & Malignant $(n=30)$ \\
\hline Presentation & Seizures & Multiple symptoms \\
Duration & Recent onset & Acute or subacute \\
Raised intracranial pressure & Absent & Present in majority \\
CT features & Parenchymal or convexity & Basal, cisternal and ventricular \\
CSF pleocytosis & leptomeningeal lesions & cysts \\
CSF protein & Infrequent & Present in all \\
CSF glucose & Normal & Increased in most \\
Hydrocephalus & Normal & Decreased in most \\
Surgery & Absent & Present in majority \\
Mortality & Seldom required & Required in majority \\
Morbidity & Nil & High \\
\hline
\end{tabular}

*Multiple symptoms as shown in table 2 .

The benign group consisted of 70 patients of whom 58 presented with epilepsy only, the majority having grand mal seizures. In this group cysts on radiological investigation were found to lie within the brain parenchyma or the convexity leptomeninges and could easily be quantitated. Pleocytosis was not detected in CSF samples and these patients had no neurological disability-their epilepsy being easily controlled with appropriate medication. A further 12 patients included in this benign group were diagnosed incidentally as having cerebral cysticercosis when cysts were observed in brain scans performed for reasons other than cysticercosis. Of these 12 patients, 11 were investigated because of recent head injuries, and the remaining patient presented with a fluctuating visual scotoma due to a single cysticercal cyst in the vitreous.

Sera and CSF were obtained from patients who had given their informed consent previously.

\section{Controls}

Included in this study were one hundred white urban individuals and 100 South African black patients living in a rural setting, whose sera were referred to this Institute for investigations not associated with cysticercosis. In addition, sera were obtained from $\mathbf{5 0}$ individuals with other helminthic infections. This group comprised of patients infected with the following parasites: Echinococcus granulosus (20), Wuchereria bancrofti (8), Schistosoma mansoni (10) and Toxocara canis (12). The diagnoses of these patients were confirmed by clinical, parasitological and serological data.

Table 2 Complex neurological features seen in malignant neurocysticercosis $(n=30)^{\star}$

\begin{tabular}{lc}
\hline Major pathological features & $\begin{array}{l}\text { Number of } \\
\text { patients }\end{array}$ \\
\hline Stupor/coma & 6 \\
Mental change/confusion & 5 \\
Hemiparesis & 4 \\
Dysphasia & 3 \\
Ataxia & 6 \\
Headache/meningism & 12 \\
Visual loss/optic atrophy & 2 \\
Papilloedema & 8 \\
Abdrucens paresis & 4 \\
Parinaud's syndrome & 3 \\
Epilpsy with other neurological disorder & 10 \\
\hline
\end{tabular}

*Patients demonstrate one or more of these major features.
Classification and quantitation of cysts on radiological examination

The position and the number of cysts were easily determined on brain scan as described by Farrell. ${ }^{9}$ Four stages of cyst development could be identified. Vesicular cysts appeared as non-enhancing lucencies in the substance or over the surface of the brain. The later stage colloidal cysts showed ring enhancement with contrast media cysts whereas the granular-nodular cyst were smaller lesions, which enhanced in a homogeneous manner. The most advanced nodular calcified cysts were identfied as calcified foci without any enhancement.

\section{Preparation of antigen}

Pigs naturally infected with cysticercosis were supplied by the Johannesburg abattoir. Cysts of approximate diameter $10 \times 12 \mathrm{~mm}$ which had a clear fresh appearance were excised from either tongue or chest wall muscles and washed twice in saline after which cyst fluid was collected using a tuberculin syringe. The protein concentration in the fluid was estimated and standardised at a level of $20 \mathrm{mg} / \mathrm{ml}$ of protein using sterile saline. Cyst fluid was pooled and stored at $-70^{\circ} \mathrm{C}$ until utilised in the ELISA.

Enzyme-linked immunosorbent assay (ELISA) The indirect micro-ELISA was carried out by the method used by Voller et al, ${ }^{10}$ using high binding capacity detachable microwells (Nunc, Denmark). The antigen concentration used to sensitise the plates was determined by a previous checkerboard titration and used at a dilution of 1:3000. Wells were coated with

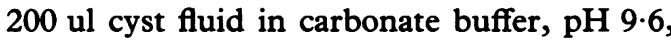
and the plates incubated for 12 hours at $4^{\circ} \mathrm{C}$. The wells were then washed with phosphate buffered saline, $\mathrm{pH} 7 \cdot 2$, containing $0.05 \%$ Tween 20 (PBS-Tween). Plates not used immediately were frozen at $-70^{\circ} \mathrm{C}$ until used. Two hundred millilitres of sera, diluted at 1:100 and CSF at 1:10 in PBS-Tween were added to wells and the plates incubated at room temperature for two hours.

Alkaline phosphatase conjugated rabbit anti-human IgG (MA Bioproducts, Maryland) at a working dilution of 1:4000 (200 ul) was added to the each well and the plates incubated for a further two hours. After a further wash, substrate solution consisting of $40 \%$ para-nitrophenyl-phosphate diluted in diethanolamine buffer (MA Bioproducts, Maryland) was added to the wells (20 ul/well) and the plates incubated for 20 minutes at room temperature. The reaction was terminated by the addition of $50 \mathrm{ul}$ of $3 \mathrm{~N} \mathrm{NaOH}$ and plates were read in a Titertek multiscanner at $405 \mathrm{~nm}$.

\section{Results}

ELISA values in various clinical groups

Sera from urban controls demonstrated OD readings of less than $0 \cdot 1$ (group $A$ in fig 1 ). Of the 50 patients with known infections by tissue invasive parasites other than $T$ solium, sera 


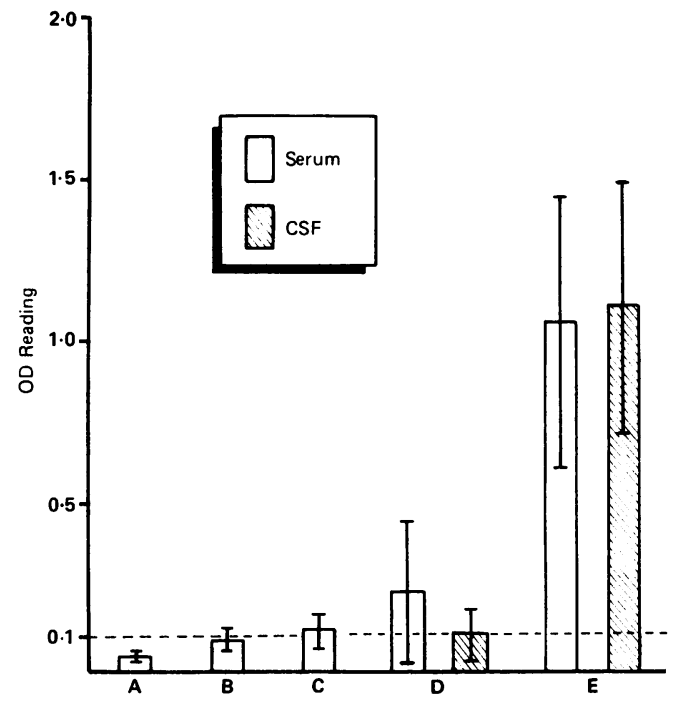

Figure 1 ELISA results of serum and CSF from: $A$ White urban controls

$B$ Rural patients with other infections

$C$ Black rural controls

$D$ Patients with benign cysticercosis

E Patients with malignant cysticercosis.

from all but two demonstrated readings of less than $0 \cdot 1$ (fig 1B). These patients were subsequently diagnosed as suffering from Echinococcus granulosus and Toxocara canis infections (confirmed by the Behring haemaglutination test and ELISA respectively). Sera from 12 of the 100 rural controls demonstrated OD readings of greater than 0.1 with a mean OD value of 0.101 (fig 1C). Sera from patients with benign cysticercosis demonstrated a mean OD reading of 0.230 (fig $1 \mathrm{D}$ ). OD levels of $<0.1$ were recorded in 14 of the 70 from this group. Interestingly 12 of these 14 with low serum OD readings were identified incidentally and had no symptoms of cerebral cysticercosis. In con-

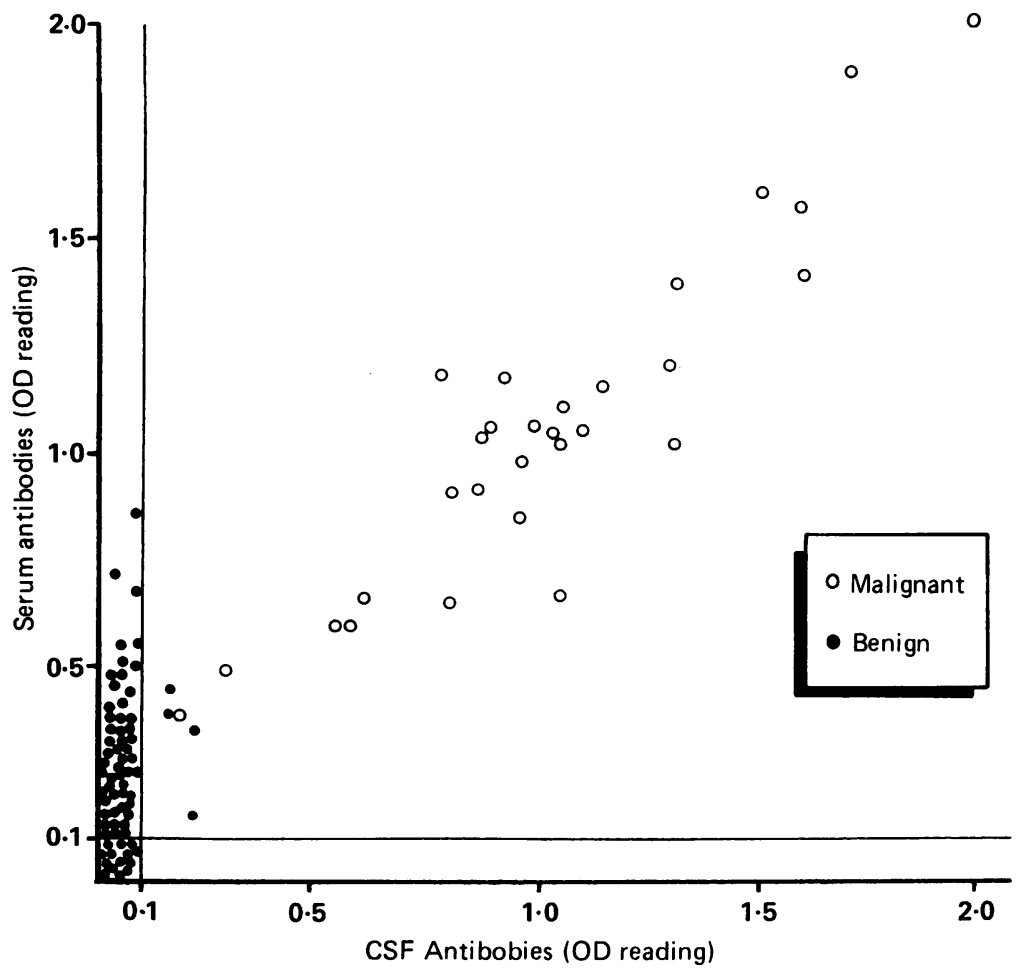

Figure 2 Antibody levels in serum and CSF from patients with benign and malignant cysticercosis. trast, sera from all the patients with malignant cysticercosis demonstrated raised antibody levels (mean OD 1.050) (fig 1E).

When CSF from patients with cysticercosis was used in the ELISA, marked differentiation between the two groups of patients was evident (fig $1 \mathrm{D}$ and $\mathrm{E}$-shaded). The CSF from patients with malignant cysticercosis had significantly elevated levels of antibodies (mean $1 \cdot 10$ ) (fig $1 \mathrm{E}$ ). In contrast, CSF from patients with the benign form of cysticercosis provided low levels of antibody to cysticercal antigens (fig 1D). In this group of patients, however, CSF from four individuals demonstrated OD levels greater than $0 \cdot 1$ (mean $0 \cdot 185$, range $0 \cdot 15$ $0 \cdot 22$ ).

Correlation of lymphocyte count and antibody levels

CSF pleocytosis was only observed in the group of patients with malignant cysticercosis (results not shown). Within this group no correlation could be detected between the actual lymphocyte count in the CSF and the antibody levels in either the serum or the CSF. Whereas some patients with high lymphocyte CSF counts had low CSF or serum antibody titres, other patients had high antibody levels. Similarly some patients with low lymphocyte counts had high antibody levels, whereas others had low levels in both the serum and the CSF.

Correlation of antibody levels in sera and CSF

The levels of antibody in the serum of patients with malignant cysticercosis correlated with antibody levels of the CSF of these individuals (fig 2, Pearson's correlation coefficient: 0.8242; $\mathrm{p}<0.0001)$. However, no correlation in antibody levels between the serum and CSF of patients with benign cysticercosis was found. A number of these patients demonstrated raised serum antibody levels with low antibody titres in their CSF.

Serum and CSF antibody levels in patients with different types of cysts detected by brain scan Upon brain scanning 100 patients, 36 individuals were seen to have calcified cysts, 27 had active cysts ( 12 colloidal and 15 vesicular) and the remaining 37 had mixed cysts. When the levels of anti-cysticercal antibodies in the serum and CSF of these patients were assessed, no relationship between the antibody level and the type of cyst could be determined. Furthermore, raised antibody levels in CSF and serum seen in patients with malignant cysticercosis, was independent of the type of cysts observed. Similarly, the low antibody titres observed in the benign group were related to the type of cysts.

Antibody levels related to the number of cysts When the number of cysts were counted on brain scans from patients with malignant cysticercosis and the cyst counts compared to serum antibody titres, no correlation was observed (fig 3). However, when the same comparisons were made using the benign group, a signficant correlation between the 


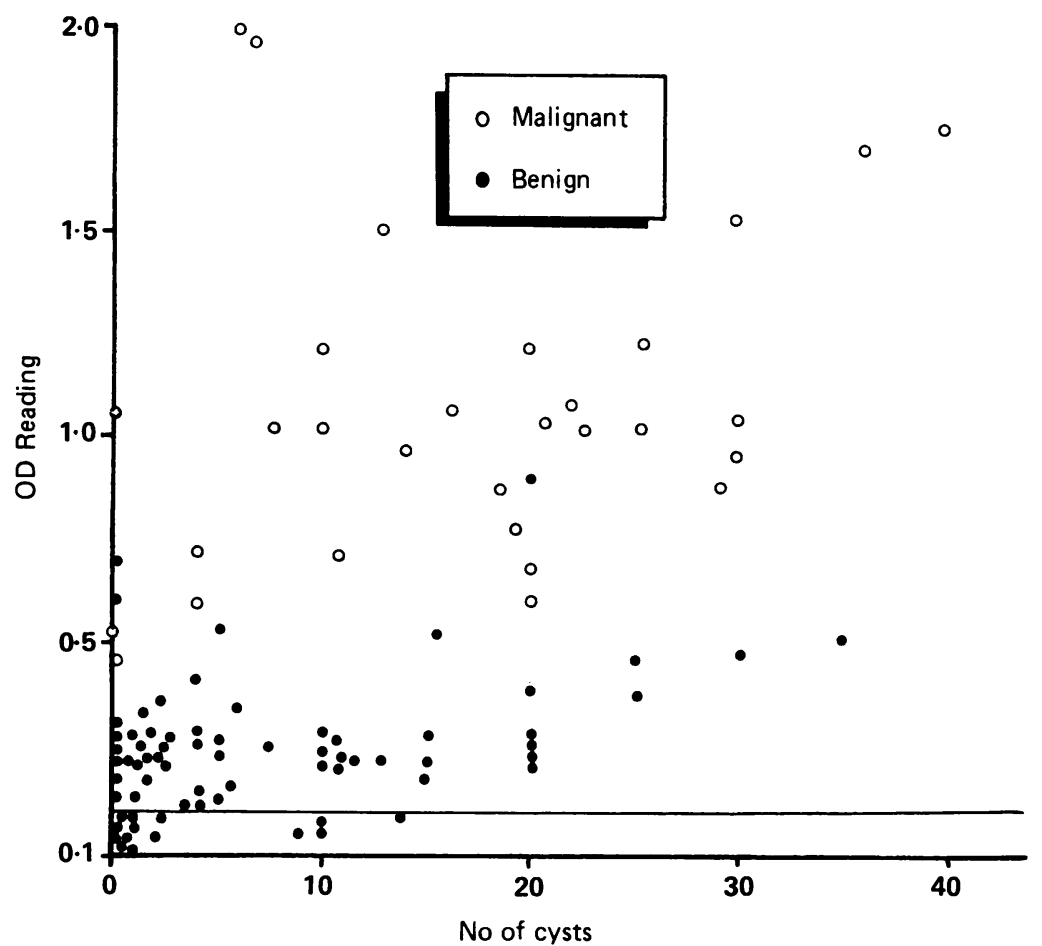

Figure 3 Relationship of the number of cysts observed on brain scan to serum and CSF antibody levels.

Figure 4 Antibody levels in patients with benign or malignant cysticercosis related to the position of cysts in the brain: $A$ cerebral cortex $B$ ventricle

$C$ base of brain number of cysts and antibody levels was observed (Pearson's correlation coefficient: $0.3582 \mathrm{p}<0.01)$.

Antibody levels related to the anatomical position of the cysts

Significant antibody titres in the CSF were only observed in those patients with cysts situated at the base of the brain or in the major cisterns or within the ventricles (fig $4 B, C$ ). Those patients with cysts in the brain cortex generally had low antibody levels, and comprised mainly of patients with benign cysticercosis (fig 4A).

\section{Discussion}

Cerebral cysticercosis is a highly heterogenous and complex disease and has been classified into benign and malignant forms. ${ }^{11}$ In our study the benign form of the disease included patients with parenchymal and calcified cysts with few or no symptoms. The malignant group comprised patients with hydrocephalus, vasculitis and cerebral infarction, associated with multiple granulomata or giant supra ventricular cysts. ${ }^{11}$

Our study employed this clinical classification rather than the stage of cyst development to differentiate the two groups of patients with cerebral cysticercosis. The results indicates that the ELISA confirms this classification in the majority of patients. All patients with the malignant form had considerably raised antibody titres in both serum and CSF. Most patients with the benign form had low or negative ELISA results in the serum and CSF (fig 1). Even though it is possible that extracerebral cysts present in some patients in the latter group may have influenced antibody levels, our results suggest that high antibody levels in the serum or the presence of antibodies in the CSF is indicative of the malignant form of cysticercosis.

A number of workers have utilised a variety of serological techniques to detect antibodies in the serum and CSF of patients with cerebral cysticercosis. ${ }^{23}$ Most of these techniques showed lack of specificity. In particular, cross reactions with various forms of other Taenia species and tissue parasites have been noted. ${ }^{12} 13$

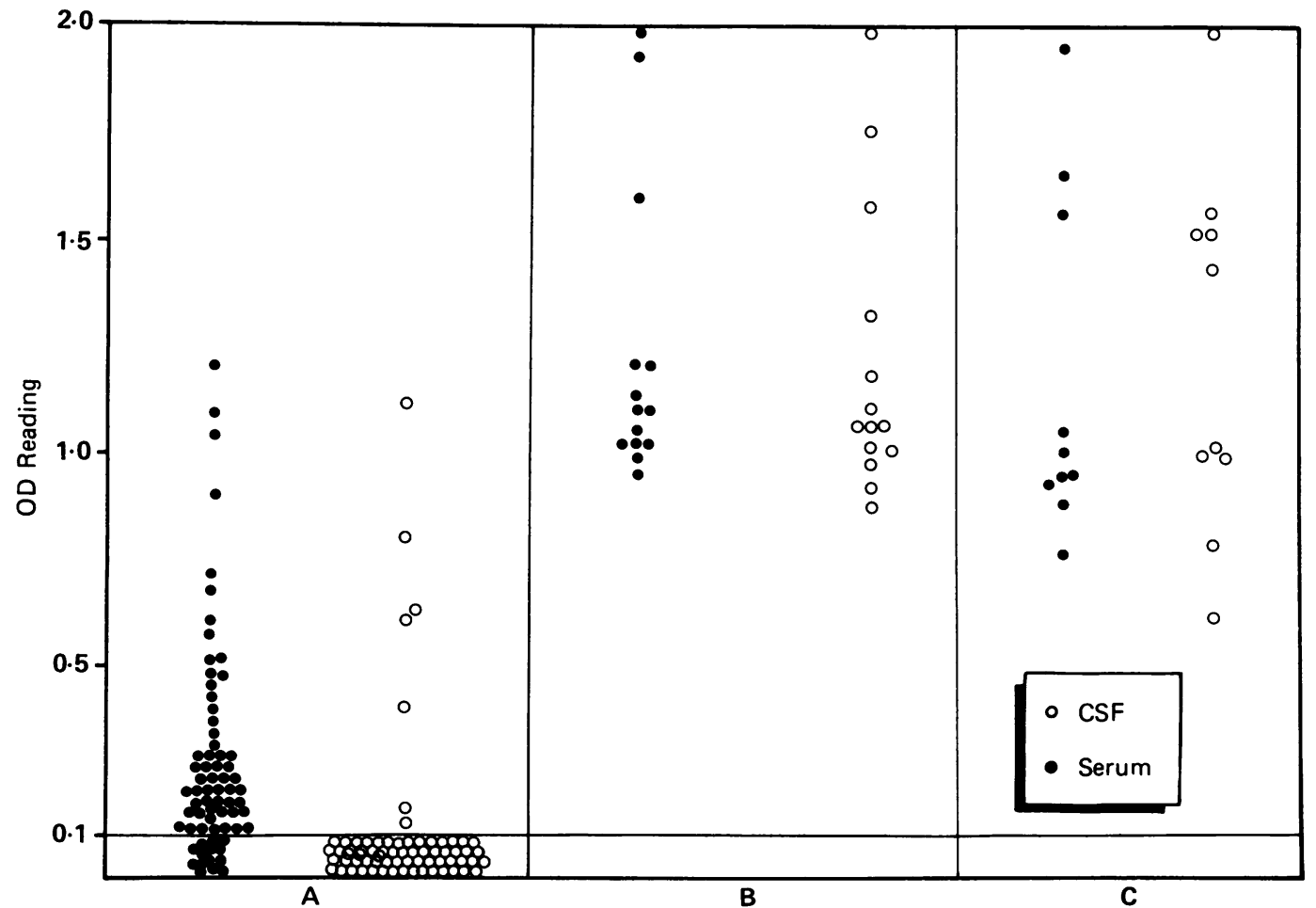


Employing cyst fluid as antigen, our study showed weakly positive reactions in only one patient with echinococcus infection and in one with a toxocara infection. Since cyst fluid contains the metabolic products of the organism, it was employed in this study to provide high specificity for the ELISA. Other workers have also demonstrated highly specific results when similar products from other parasites were used in ELISA. ${ }^{14}$ Furthermore, the use of cyst fluid in ELISA has also recently been documented to provide reliable serological data for the diagnosis of cysticercosis ${ }^{1516}$ and also indicate greater specificity when compared to studies using whole cysticercal antigen preparations. ${ }^{16}$

An interesting feature of this study was the observation that a number of rural patients with non-parasitic disease nevertheless had positive antibody levels as demonstrated by the ELISA for cysticercosis. This presumably indicates a subclinical form of infection common in Africa. It was interesting that 12 patients in the benign group were detected purely incidentally while being investigated for other problems. As expected, urban dwellers had very low levels of cysticercus antibody.

This study also examined whether lymphocyte counts in the CSF had any relationship with antibody levels in the serum or CSF. The results indicate the absence of any relationhsip between lymphocyte counts and serum or CSF antibody levels in patients with malignant cysticercosis. It is unclear why some patients with the malignant disease who have high lymphocyte counts had minimal antibody levels whereas others with low lymphocyte counts showed high serum or CSF antibody levels. Presumably the lymphocyte response reflects local pathology and the presence of meningitis rather than changes in the specific immune response. In addition, our study also found that all the patients with the benign form of the disease lacked both significant numbers of lymphocytes (results not shown) and lower antibody levels in the CSF.

The results of antibody levels in the CSF are comparable to those described in a recent study from Mexico. ${ }^{11}$ Patients with malignant cerebral cysticercosis were shown to have correspondingly high levels of antibodies both in the serum and the CSF, and the higher the levels in the serum, the greater the antibody titres in the CSF. In contrast, patients with the benign form of cysticercosis had detectable antibodies only in the serum, and these were generally at a lower titre than those of the patients with malignant cysticercosis. The reason for the relatively low antibody titres in patients with benign cysticercosis is unclear but Corona et al ${ }^{11}$ suggested that patients with malignant cerebral cysticercosis have a much more marked humoral response than those with a benign course.

It has been suggested that the serological response in cysticercosis may depend upon the stage of development of the cyst. ${ }^{7}$ This study, however, demonstrates that cyst development as observed on brain scan was not related to antibody levels. Some patients who had cal- cified cysts showed high antibody titres, while other patients with low antibody levels showed the presence of either active or calcified cysts. Rather antibody levels appeared to be related to the clinical differentiation of cysticercosis. Raised antibody levels were related to malignant cysticercosis and low antibody levels seen in the benign group of cysticercosis.

In the malignant cysticercosis group, not only was there no relationship between antibody levels and stage of cyst development, but the number of cysts observed on brain scan did not influence the humoral response. Some patients with very few brain cysts had in some instances very high antibody levels (fig 3). Antibody levels, however, appeared to be correlated to the number of cysts observed on brain scans of benign cysticercosis patients (correlation coefficient 0.358 ). Cerebral cysticercosis forms only part of a much more generalised disease with multiple organ involvement and it is not altogether surprising therefore that antibody levels do not always relate to the number of cerebral cysts.

A further aspect of this study investigated the relationship of antibody levels to the position of the cysts in the brain. Most patients with the benign form of the disease had cysts located in the brain cortex and these were generally associated with a poor humoral response (fig 4). Patients with lesions in the brain stem, the ventricles or cisterns, however, had the highest antibody levels and these were usually patients with the malignant form of the disease (fig 4). Although the reason for the high antibody response in patients with cysts in certain anatomic regions is unclear, it is possible that these sites may represent areas where the blood-brain barrier is not intact and the lymphocytes may have access to the brain. ${ }^{17}$ Oncospheres reach the brain via the blood circulation and in the case of the cerebral hemispheres this is such that the oncospheres tend to lodge at the junction of the grey and the white matter. At this site they are distant from the meninges and the CSF and little immunological response is evoked. If the cysts, however, lodge in close proximity to the ventricular system or meninges, a more intense immune reaction associated with pleocytosis results, and these patients follow a more aggressive clinical course.

These studies indicate that ELISA can be used effectively to differentiate between benign and malignant cysticercosis. Significant correlation exists between antibody levels found in the sera and CSF of patients with malignant cysticercosis whilst antibody levels are related to the number of cysts in the benign group only. Furthermore, antibody levels appear to be related to the anatomical position of cysts but are not related to lymphocyte counts in the CSF or the type of cysts seen on brain scans.

This work was partly supported by grants from the Medical Research Council of South Africa and the South African Institute for Medical Research, Johannesburg. We are grateful to the Superintendent, Rand Mutual Hospital, Johannesburg, for the use of case material. 
1 Powell SJ, Proctor EM, Wilmot AJ, Barnett AM. Neurological complication of cysticercosis in Africans-a
clinical and serological study. Ann Trop Med Parasitol clinical and sero

2 Shasha W, van Dellen J, Cakata E. Cysticercosis: an analysis of 141 cases in South Africa. S Afr J Epidemiol Infect 1986;1:94-7.

3 Camargo CA, Marshall WH. Radiological diagnosis of neurocysticercosis. Parasitol Today 1987;3:30-1.

4 Diwan AR, Cocker-Vann M, Brown P, et al. Enzyme-linked immunosorbent assay (ELISA) for the detection of ntibody to cysticerci of Taenia solium. Am J Trop Med Hyg 1982;31:364-9.

5 Costa JM, Ferreira AW, Makiro MM, Camarango ME. Spinal fluid immunoenzymatic assay (ELISA) for neurocysticercosis. Rev Inst Med Trop Sao Paulo 1982; neurocysticerce

6 Gottstein B, Tsang VCW, Schantz PM. Demonstration of species-specific and cross-reactive components of Taenia solium metacestodes antigen. Am J Trop Med Hyg 1986; 35:308-13.

7 Schantz PM, Shanks D, Wilson M. Serological cross-reactions with sera from patients with echinococcosis and cysticercosis. Am J Trop Med Hyg 1980;29:609-12.

8 Espinoza B, Flisser A, Plancarte A, Larralde C. In: Flisser A, et al, eds. Cysticercosis: present state of 1201630 and

9 Farrell VJR. Cerebral Cysticercosis: a pathological, radiological and clinical study with comments on treatment. Hunterian Lecture: RCS (London) 1987. Ann
Royal College of Surgeons (in press).

10 Voller A, Bidwell DE, Barlett A. Enzyme immunoassay in diagnostic medicine: theory and practice. Bull WHO diagnostic

11 Corona T, Pascoe D, Gonzales-Barranco D, et al. Anticysticercous antibodies in serum and cerebro-spinal fluid in patients with cerebral cysticercosis. J Neurol Psychiatry 1986;49:1044-9.

12 Flisser A, Larralde C. Cysticercosis. In: Walls KW, Schantz PM, eds. Helminthic diseases; parastic diseases. Florida London: Acad Press, 1986;109-61.

13 Gottstein B, Zini D, Schantz PM. Species-specific immunodiagnosis of Taenia solium cysticercosis by 38:299-303.

14 De Savigny DH, Voller A. ELISA for toxocariosis using larval secretory antigens. Trans $R$ Soc Trop Med Hyg

15 Larralde C, Laclette JP, Owen CS, et al. Reliable serology of Taenia solium cysticercosis with antigens from cyst vesicular fluid: ELISA and hemagglutination tests. $A m \mathrm{~J}$ Trop Med Hyg 1986;35:965-73.

16 Baily GG, Mason PR, Trijssenar FEJ, Lyons NF. Serological diagnosis of neurocysticercosis: evaluation of ELISA tests using cyst fluid and other components of Taenia solium cysticerci as antigens. Tran Roy Soc Trop Med Hyg 1988;82:295-9.

17 Link H. Some aspects of immune reactions of the brain. In: F Clifford Rose, ed. Clinical Neuroimmunology. Oxford: Blackwell Scientific Publications, 1979:12-28. 